\title{
Association of NOS3 gene variants and clinical contributors of hypoxic-ischemic encephalopathy
}

\author{
R. Kuzmanić Šamija ${ }^{1}$, D. Primorac ${ }^{2,3,4,5}$, B. Rešić ${ }^{2}$, V. Pavlov ${ }^{6}$, V. Čapkun ${ }^{7}$, H. Punda ${ }^{2}$, \\ B. Lozić ${ }^{1}$ and T. Zemunik \\ ${ }^{1}$ Department of Pediatrics, University Hospital Split, Split, Croatia \\ ${ }^{2}$ School of Medicine Split, University of Split, Split, Croatia \\ ${ }^{3}$ Department of Pediatrics, School of Medicine, University of Osijek, Osijek, Croatia \\ ${ }^{4}$ Eberly College of Science, Penn State University, University Park, PA, USA \\ ${ }^{5}$ St. Catherine Speciality Hospital, Zabok, Croatia \\ ${ }^{6}$ Department of Neonatology, University Hospital Split, Split, Croatia \\ ${ }^{7}$ Department of Nuclear Medicine, University Hospital Split, Split, Croatia \\ ${ }^{8}$ Department of Medical Biology, School of Medicine Split, University of Split, Split, Croatia
}

\begin{abstract}
The aim of this study was to analyze the association of different clinical contributors of hypoxic-ischemic encephalopathy with NOS3 gene polymorphisms. A total of 110 children with hypoxic-ischemic encephalopathy and 128 control children were selected for this study. Association of gender, gestational age, birth weight, Apgar score, cranial ultrasonography, and magnetic resonance imaging findings with genotypic data of six haplotype-tagging single nucleotide polymorphisms and the most commonly investigated rs1800779 and rs2070744 polymorphisms was analyzed. The TGT haplotype of rs1800783, rs1800779, and rs2070744 polymorphisms was associated with hypoxic-ischemic encephalopathy. Children with the TGT haplotype were infants below 32 weeks of gestation and they had the most severe brain damage. Increased incidence of the TT genotype of the NOS3 rs1808593 SNP was found in the group of hypoxic-ischemic encephalopathy patients with medium and severe brain damage. The probability of brain damage was twice as high in children with the TT genotype than in children with the TG genotype of the same polymorphism. Furthermore, the T allele of the same polymorphism was twice as frequent in children with lower Apgar scores. This study strongly suggests associations of NOS3 gene polymorphism with intensity of brain damage and severity of the clinical picture in affected children.
\end{abstract}

Key words: Clinical contributors; Hypoxic-ischemic encephalopathy; NOS3 gene polymorphisms

\section{Introduction}

Hypoxic-ischemic encephalopathy (HIE), a serious condition affecting infants in the perinatal period, is the most common cause of neurologic deficits in children. It may occur in premature and full-term children as a result of an intrauterine infection and/or chorioamnionitis, premature rupture of membranes, maternal trauma and shock (1). Lack of oxygen, caused by impairment of cerebral blood flow, starts a cascade of harmful biochemical processes at the cellular level, resulting in serious brain damage (2-4)

One of the mechanisms of cell-structure degradation is mediated by activation of a nitric oxide synthase (NOS) and by formation of free radicals of oxygen and nitrogen (5-7). Exposure to mild ischemia activates endothelial nitric oxide synthase (NOS3), a $\mathrm{Ca}^{2+}$-dependent enzyme; small amounts of NO are produced, causing vasodilatation, and the consequent maintenance of cerebral blood flow prevents neuronal injury. NO catalyzed by NOS3 also affects other processes such as inhibition of platelet and leukocyte adhesion to endothelium, inhibition of platelet aggregation, relaxation and inhibition of cell proliferation of blood vessel smooth muscle, stimulation of angiogenesis, anti-inflammatory action, and prevention of oxidative damage (8). Production of NO in endothelial blood vessel cells is regulated by the NOS3 gene (OMIM: 163729). Several studies reported that NOS3 gene polymorphisms may alter enzymatic function and therefore may be associated with HIE (9-11).

Our previous study showed genotype and haplotype association of NOS3 gene polymorphisms with HIE. 
Genotypic tests of six haplotype-tagging single nucleotide polymorphisms (SNPs; rs3918186, rs3918188, rs1800783, rs1808593, rs3918227, rs1799983), and the most commonly investigated rs 1800779 SNP, showed an association of rs1808593 polymorphism with HIE. The TG genotype of rs1800783-rs1800779 polymorphisms was found less frequently, and the TT genotype more frequently, in a group of affected patients than in a control group. We also observed an association of the TG haplotype with HIE (12).

Severity of clinical presentation and degree of brain damage can be influenced by a genetic predisposition to development of the disease. The aim of this study was to analyze the association of different clinical contributors that define the severity of disease and patient condition with NOS3 gene polymorphisms that we investigated in our previous study.

\section{Material and Methods}

A total of 110 unrelated full-term or preterm children (69 boys and 41 girls) with HIE (HIE group) were recruited for the study. The study included all HIE patients treated in the University Hospital Split, Croatia between 1992 and 2008, and included children who met at least one of the following criteria: fetal heart rate during delivery $<100$ or $>160 / \mathrm{min}$, Apgar score $\leq 7$ at $5 \mathrm{~min}$, arterial $\mathrm{pH} \leq 7.1$, neonatal neurological sequelae classified by Sarnat and Sarnat (13), or positive hypoxic-ischemic brain damage found on repeated cranial ultrasonography at different time periods. Perinatal hypoxic-ischemic brain injuries of newborns were detected by cranial ultrasonography. With cranial ultrasonography, we determined the grade of periventricular leukomalacia (PVL) according to criteria described by Weisglas-Kuperus (14): grade I PVL, prolonged periventricular flare present for 7 days or more; grade II PVL, prolonged periventricular flare with small, localized frontoparietal cysts; grade III PVL, extensive periventricular cystic lesions involving occipital and frontoparietal white matter; grade IV PVL, areas of extensive subcortical cystic lesions.

Hypoxic-ischemic brain damage in full-term infants was also confirmed by serial cranial ultrasonography performed at 3, 7, 14, and 21 days after delivery, followed by once per month until closing the anterior fontanel. Ultrasonography was performed by two independent experts.

Final diagnosis and grade of hypoxic-ischemic brain damage in the HIE group was determined by magnetic resonance imaging (MRI) at the age of 2 years, when the processes of proliferation, migration, organization, and myelination were completed. The altered signal of the white matter, appearance of cystic changes, changes in the size of the lateral ventricle anterior horns, extension of subarachnoid space and thinning of the corpus callosum were followed (Table 1) $(15,16)$. Each parameter that was observed was scored from 1-3 points: grade 1, total scores of up to 5 points were considered normal findings. grade 2, moderate injury, included total scores of 610 points, and grade 3 , severe brain injury, comprised total scores of 11-15 points.

For the two groups studied, demographic data and other characteristics such as gender, gestational age ( $\geqslant 37,32$ to $36,<32$ weeks), and birth weight were collected from the medical documentation. For the data analysis, study participants were divided into four subgroups according to birth weight ( $\leq 1000,1001-1499$, 1500-2499, and $\geqslant 2500$ g.) and Apgar score ( $\leq 5,6-7,8-9$, and 10). Patients with congenital malformations of the central nervous system (CNS), metabolic diseases, chromosomal abnormalities, with infections, and patients with CNS trauma, were excluded. We also excluded infants from multiple gestations, and infants of mothers who had preeclampsia and placental abruption.

The HIE group consisted of 80 patients $(72.7 \%)$ with hypoxic-ischemic injury and 30 patients $(27.3 \%)$ with hypoxic-ischemic injury accompanied by intracranial hemorrhage. The control group consisted of 128 apparently healthy full-term and preterm born children ( 60 boys and 68 girls), with normal brain ultrasound findings and/or normal neurological status after the second year of life. The HIE and the control subject populations were the same as in our previous publication (12).

Additionally, another frequently investigated polymorphism, rs2070744, was genotyped in both the HIE and control groups. Real-time polymerase chain reaction (PCR) was performed using the ABIPRISM 7500 Sequence Detection System (Applied Biosystems, USA) following the manufacturer's protocol.

The study was approved by the Ethics Committee of University Hospital Split and School of Medicine, University of Split, and informed consent was obtained from patients' parents prior to the blood sampling.

\section{Statistical analysis}

Quality control analysis of the obtained rs2070744 genotypes was performed. Genotype frequencies, HardyWeinberg equilibrium (HWE), and minor allele frequencies (MAF) for all samples were analyzed using HaploView 4.1 (http://haploview.software.informer.com/4. $1 /$ ). An $80 \%$ study power at $\alpha=0.05$ was calculated using Quanto (http://biostats.usc.edu/Quanto.html), and results are reported as odds ratios (ORs). When $\mathrm{OR}>1$, the genotype confers sensitivity to the effects of exposure (17). Case-control association analysis as well as cisposition haplotype analysis of 8 SNPs was carried out using Haploview 4.1.

Chi-square tests were used to analyze genotypic and allelic distribution for each polymorphism examined by gestational age, gender, Apgar score, birth weight, and MRI findings. The Yates correction was performed when necessary. 
Table 1. Scoring of perinatal hypoxic-ischemic brain injury according to magnetic resonance imaging.

\begin{tabular}{|c|c|c|c|}
\hline & $\begin{array}{c}\text { Grade } 1 \\
\text { (normal findings) }\end{array}$ & $\begin{array}{l}\text { Grade } 2 \\
\text { (moderate brain injury) }\end{array}$ & $\begin{array}{c}\text { Grade } 3 \\
\text { (severe brain injury) }\end{array}$ \\
\hline $\begin{array}{l}\text { White matter signal } \\
\text { abnormality }\end{array}$ & Normal signal & Focal altered signal (one region) & $\begin{array}{c}\text { Changed signal to } 2 \text { or more } \\
\text { regions }\end{array}$ \\
\hline $\begin{array}{l}\text { Size of lateral ventricle } \\
\text { anterior horns }\end{array}$ & Proper size up to $8 \mathrm{~mm}$ & Mild to moderate dilatation, $8-10 \mathrm{~mm}$ & Significant dilatation $>10 \mathrm{~mm}$ \\
\hline Cystic change & No & Focal cystic changes & $\begin{array}{c}\text { Extensive cystic changes in white } \\
\text { matter }\end{array}$ \\
\hline Corpus callosal thinning & Normal & Thinned $<3 \mathrm{~mm}$ focal & Thinned $<3 \mathrm{~mm}$ multifocal \\
\hline Subarachnoid space & Proper size up to $4 \mathrm{~mm}$ & Slightly extended, 4-8 mm & Moderate to very extended $\geqslant 8 \mathrm{~mm}$ \\
\hline
\end{tabular}

Logistic regression (Statistica 7.0; http://statistica. software.informer.com/7.0/) was performed to calculate $\mathrm{OR}$ in order to determine incidence of hypoxic-ischemic brain damage with respect to gestational age, gender, Apgar score, birth weight, and haplotype. Multiple logistic regression with regard to haplotype distribution was also performed in order to prove logistic regression results. Chi-square tests were also performed to compare the degree of brain damage established by MRI with regard to gender, gestational age, birth weight, and Apgar score.

\section{Results}

The genotype call rate for the investigated rs2070744 polymorphisms was $100 \%$, and the genotypes in the case and control groups fit HWE. The rs2070744 polymorphisms showed strong linkage disequilibrium with earlier investigated rs1800783 and rs1800779 polymorphisms $\left(r^{2}=0.96\right.$ and $r^{2}=0.99$, respectively). Allelic and genotype analysis of rs2070744 polymorphism did not show any association with HIE. The study had $80 \%$ statistical power to detect $(\alpha=0.05)$ an effect with an OR $=1.76$ for rs2070744, assuming an additive model. In addition, the cis TGT haplotype of rs1800783, rs1800779, and rs2070744 polymorphisms of the NOS3 gene was found in only five of the HIE patients and was not found in the control group.

Genotype distribution analysis of the six haplotypetagging SNPs (rs3918186, rs3918188, rs1800783, rs1808593, rs3918227, rs1799983) and the most commonly investigated rs1800779 and rs2070744 SNPs were determined in the case and control groups and evaluated according to clinical contributors (i.e., gestational age, gender, Apgar score, birth weight, and MRI findings). The analysis showed that the TT genotype of rs1808593 polymorphism was twice as common as TG and GG in the HIE group in patients with moderate brain injury (grade 2; $\mathrm{TT}=26, \mathrm{TG}=11, \mathrm{GG}=1$ ) and severe brain injury (grade 3; $\mathrm{TT}=41, \mathrm{TG}=25, \mathrm{GG}=6$ ) than in the control group (grade 1; $\mathrm{TT}=59, \mathrm{TG}=66, \mathrm{GG}=3 ; \chi^{2}=12.36, \mathrm{P}=0.015$ ). In children with normal MRI findings (grade 1 ), the most common rs 1808593 genotype was TG.

Comparing the genotype distribution of the same rs1808593 polymorphisms between normal MRI findings (grade 1) and pathological MRI findings regardless of the severity of damage (grades 2 and 3), a statistically significant association $\left(\chi^{2}=6.572, \mathrm{P}=0.0104\right)$ was found, with a higher incidence of the TT genotype in children with abnormal MRI findings.

The allele distribution analysis of all investigated polymorphisms showed a statistically significant more frequent occurrence of the $T$ allele of the rs1808593 polymorphism in children with low Apgar scores. The $T$ allele was more frequent in all groups, as expected; but the difference between $T$ and $G$ allele frequency was more significant as Apgar score was lower $\left(\chi^{2}=10.4\right.$ $\mathrm{P}=0.015$; Table 2).

The genotype distribution analysis of polymorphism rs3918186, showed that AA was twice as frequent as AT in children with normal Apgar scores $(A S=10)$ but that the difference was not statistically significant $\left(\chi^{2}=9.4\right.$, $\mathrm{P}=0.152$; Table 3). Furthermore, analysis of HIE children with TGT haplotype polymorphisms rs 1800783, rs1800779, rs2070744 of the NOS3 gene showed that they were premature infants of $<32$ weeks of gestation with the most severe degree of brain damage (grade 3 ) on the MRI findings.

Logistic regression analysis showed that the OR for incidence of hypoxic-ischemic brain damage was up to two times higher in male than in female children $(P=0.01$; Table 4). The OR for the occurrence of disease was 1.92 times higher in children with Apgar scores $<5$ than in those with scores of $10(P<0.001)$. Also, the OR for the occurrence of disease was 1.32 times higher in children with lower birth weight $(P=0.03)$ and 1.62 times higher in children with lower gestational age $(P=0.003)$. Since the TGT haplotype was found in only five patients with $\mathrm{HIE}$, logistic regression analysis was performed only for the two predominant haplotype combinations, TAT and AGC, and showed no significant difference in occurrence $(P=0.573$; Table 4).

After applying multiple logistic regression with regard to haplotype distribution, the difference in occurrence of 
Table 2. Allele distribution analysis of NOS3 rs1808593 polymorphism and Apgar score.

\begin{tabular}{lrrrrr}
\hline Apgar score & Total alleles & T allele & G allele & $\chi^{2}$ & $P$ \\
\hline$\leq 5$ & $166(100 \%)$ & $130(78 \%)$ & $36(22 \%)$ & 10.4 & 0.015 \\
$6-7$ & $142(100 \%)$ & $105(74 \%)$ & $37(26 \%)$ & & \\
$8-9$ & $70(100 \%)$ & $45(64 \%)$ & $25(36 \%)$ & & \\
10 & $98(100 \%)$ & $74(75 \%)$ & $44(45 \%)$ & & \\
\hline
\end{tabular}

Data are reported as number with percent in parentheses. The $\chi^{2}$ test was used for analyses.

disease was maintained for gender $(\mathrm{OR}=1.98,95 \%$ $\mathrm{Cl}=1.1-3.5, \mathrm{P}=0.021)$ and Apgar score $(\mathrm{OR}=2.13$, $95 \% \mathrm{Cl}=1.5-3.0, \mathrm{P}<0.001)$.

There was a $90 \%$ probability for children of a lower gestational age to have very severe brain damage (MRI grade 3), and for children with greater gestational age ( $\geqslant 37$ weeks) to have a $90 \%$ probability to have a milder form of damage (grade 2; Table 5).

There was a $90 \%$ probability for lower birth weight children to have severe brain damage (grade 3). Also, there was almost $90 \%$ probability for children with a birth weight $>2500 \mathrm{~g}$ to have a milder form of brain damage (grade 2, Table 6).

\section{Discussion}

The results of this study strengthen the association of NOS3 gene polymorphisms with HIE and also provides evidence of their association with certain clinical contributors and intensity of brain damage. We found the TGT haplotype of NOS3 gene polymorphisms rs1800783, rs1800779, and rs2070744 in five patients with HIE, with no such haplotype in the control group. This finding highlights even more our previous finding of an rs1800783-rs1800779 TG haplotype association with HIE (12).

$\mathrm{NO}$ is an important and well-characterized vasodilator, with homeostasis of $\mathrm{NO}$ essential in maintaining vascular tone in the systemic and cerebral circulation $(8,10)$. Several earlier studies investigating possible causes of cerebral palsy indicated that genes that are associated with vascular disease or inflammation, and with the fetal inflammatory response, may also be linked with cerebral palsy development $(18,19)$. Polymorphisms rs1800783, rs1800779, and rs2070744 are located in the upstream and promoter region of the NOS3 gene that may influence mRNA transcription and can reduce gene expression; this may further lead to impaired production or reduced bioavailability of $\mathrm{NO}$, thus causing susceptibility to severe brain injury (20). Lower levels of NOS3 expression may lead to reduced protective effects in endothelial cells, thus predisposing to the occurrence of stroke (11).

This study also showed that children with the TGT haplotype were premature infants below 32 weeks of gestational age and had the most severe brain damage (grade 3) on $\mathrm{MRI}$. It is interesting that we observed rs1808593 TT genotype association with HIE in our previous study (12). In this study, we further confirmed and elucidated the significance of this polymorphism in children affected by HIE. We found a higher incidence of the TT genotype of NOS3 rs1808593 polymorphism in those patients with medium and severe brain damage.

We analyzed the genotypic distribution of polymorphism rs1808593 with regard to MRI findings and found twice the prevalence of the TT genotype in the group of children with grade 2 and grade 3 brain injury. Thus, prevalence of the TT genotype was increased in children with abnormal MRI findings regardless of the degree of damage, while among children with normal MRI findings (control group), the TG genotype was predominant

Also, this study showed a frequent occurrence of the $\mathrm{T}$ allele of rs1808593 polymorphism in children with low Apgar scores. Although the T allele was more frequent in all groups, the difference in frequency between $T$ and $G$ allele was significant in children with low Apgar scores. Such a detailed analysis of genotypic and allelic correlations with clinical contributors allows us to suggest that

Table 3. Genotype distribution analysis of NOS3 gene polymorphism rs3918186 and Apgar score.

\begin{tabular}{lccccc}
\hline Apgar score & Genotype AA & Genotype AT & Genotype TT & $\chi^{2}$ & P \\
\hline$\leq 5$ & $65(35 \%)$ & $18(40 \%)$ & $0(0 \%)$ & 9.4 & 0.152 \\
$6-7$ & $56(30 \%)$ & $15(33 \%)$ & $0(0 \%)$ & \\
$8-9$ & $27(14 \%)$ & $7(16 \%)$ & $1(20 \%)$ & \\
10 & $40(21 \%)$ & $5(11 \%)$ & $4(80 \%)$ & \\
\hline
\end{tabular}

Data are reported as number with percent in parentheses. The $\chi^{2}$ test was used for analyses. 
Table 4. Odds ratios for occurrence of hypoxic-ischemic encephalopathy according to gender, Apgar score, body weight, gestational age and haplotype distribution.

\begin{tabular}{|c|c|c|c|c|c|c|}
\hline & Control group & HIE group & $\chi^{2}$ & $\mathrm{P}$ & OR $(95 \% \mathrm{Cl})$ & $\mathrm{P}$ \\
\hline \multicolumn{7}{|l|}{ Gender } \\
\hline M & $60(47 \%)$ & 70 (64\%) & 6.7 & 0.01 & $1.98(1.18-3.34)$ & 0.01 \\
\hline $\mathrm{F}^{*}$ & $68(53 \%)$ & $40(36 \%)$ & & & & \\
\hline \multicolumn{7}{|l|}{ Apgar score } \\
\hline$\leq 5$ & $33(26 \%)$ & $50(46 \%)$ & 29.3 & $<0.001$ & $1.92(1.48-2.5)$ & $<0.001$ \\
\hline $6-7$ & $32(25 \%)$ & $39(36 \%)$ & & & & \\
\hline $8-9$ & $21(16 \%)$ & $14(12 \%)$ & & & & \\
\hline $10^{*}$ & $42(33 \%)$ & $7(6 \%)$ & & & & \\
\hline \multicolumn{7}{|l|}{ Body weight } \\
\hline$\leq 1000$ & $12(9 \%)$ & $14(13 \%)$ & 8.9 & 0.03 & $1.32(1.0-1.7)$ & 0.03 \\
\hline $1001-1499$ & $29(23 \%)$ & $28(25 \%)$ & & & & \\
\hline $1500-2499$ & $34(27 \%)$ & $42(38 \%)$ & & & & \\
\hline$\geqslant 2500^{*}$ & $53(41 \%)$ & $26(24 \%)$ & & & & \\
\hline \multicolumn{7}{|c|}{ Gestational age } \\
\hline$\leq 31$ & $45(35 \%)$ & $52(47 \%)$ & 11.6 & 0.003 & $1.62(1.2-2.2)$ & 0.003 \\
\hline $32-36$ & $34(27 \%)$ & $38(35 \%)$ & & & & \\
\hline$\geqslant 37^{*}$ & $49(38 \%)$ & $20(18 \%)$ & & & & \\
\hline \multicolumn{7}{|l|}{ Haplotype } \\
\hline TAT & $109(86 \%)$ & $91(88 \%)$ & 0.1 & 0.713 & $1.25(0.57-2.7)$ & 0.573 \\
\hline$A G C^{*}$ & $18(14 \%)$ & $12(12 \%)$ & & & & \\
\hline
\end{tabular}

Data are reported as number with percent in parentheses. HIE: hypoxic-ischemic encephalopathy; OR: odds ratio; Cl: confidence interval. " Reference value. The $\chi^{2}$ test and logistic regression were used for analyses.

the presence of the $\mathrm{T}$ allele seems to indicate $\mathrm{a}$ susceptibility for greater brain injury, and a lower Apgar score at birth.

Polymorphism rs 1808593 is located in the twenty-third intron of the NOS3 gene. Intron polymorphisms may have a potential effect on gene transcription or mRNA stability, and thus affect gene expression, and consequently, enzyme function (21). Yoon et al. (22) showed an association of the NOS3 gene polymorphism rs1808593 with NO plasma concentration in patients with coronary artery disease. Association of G894T polymorphism (rs1799983) and 10G/ T intron-23 (rs1808593) polymorphism and their haplotype combination with childhood hypertension, especially in females, were also shown (23). On the other hand, the AA genotype of polymorphism rs3918186 was twice as prevalent among children with Apgar scores of 10 , which might indicate its protective function in brain damage.
This study also found an association of low Apgar scores $<5$ at $5 \mathrm{~min}$ after birth with hypoxic-ischemic injury, which is in line with previous findings (24). A recently published study showed that the prevalence of cerebral palsy in children with an Apgar score $<3$ was 130-times higher than in children with a score of 10 (25). Similar observations have been reported in children with extremely low birth weights $(<1000 \mathrm{~g})$, who were more likely to develop HIE than children with higher birth weights. It should be emphasized that the children of low birth weight are generally premature, as gestational age and birth weight are closely correlated (26).

We also found that boys had a higher risk of HIE than girls, which is consistent with the observations of other authors. Thorngren-Jerneck et al. (27) also reported that boys have a higher risk of developing cerebral palsy than girls (1.36:1), especially if they were born prematurely

Table 5. Magnetic resonance imaging findings according to the gestational age of children with hypoxic-ischemic encephalopathy.

\begin{tabular}{lcccccc}
\hline & GA $<32$ & GA 32-36 & GA $\geqslant 37$ & Total & $\chi^{2}$ & P \\
\hline MRI grade 2 & $13(34 \%)$ & $15(40 \%)$ & $10(26 \%)$ & $38(100 \%)$ & 4.614 & 0.099 \\
MRI grade 3 & $39(54 \%)$ & $23(32 \%)$ & $10(14 \%)$ & $72(100 \%)$ & & \\
Total & $52(47 \%)$ & $38(35 \%)$ & $20(18 \%)$ & $110(100 \%)$ & & \\
\hline
\end{tabular}

Data are reported as number with percent in parentheses. GA: gestational age; MRI: magnetic resonance imaging. The $\chi^{2}$ test was used for analyses. 
Table 6. Magnetic resonance imaging findings according to the birth weight in children with hypoxic-ischemic encephalopathy.

\begin{tabular}{lrrrrrrr}
\hline & $\leq 1000 \mathrm{~g}$ & $1001-1499 \mathrm{~g}$ & $1500-2499 \mathrm{~g}$ & $\geqslant 2500 \mathrm{~g}$ & Total & $\chi^{2}$ & $\mathrm{P}$ \\
\hline MRI grade 2 & $4(10 \%)$ & $8(21 \%)$ & $12(32 \%)$ & $14(37 \%)$ & $38(100 \%)$ & 4.15 & 0.246 \\
MRI grade 3 & $10(14 \%)$ & $20(28 \%)$ & $30(42 \%)$ & $12(16 \%)$ & $72(100 \%)$ & \\
Total & $14(13 \%)$ & $28(25 \%)$ & $42(38 \%)$ & $26(24 \%)$ & $110(100 \%)$ & \\
\hline
\end{tabular}

Data are reported as number with percent in parentheses. MRI: magnetic resonance imaging. The $\chi^{2}$ test was used for analyses.

(1.55:1). Another epidemiological study showed up to $30 \%$ higher incidence of cerebral palsy among boys than girls. The risk of developing cerebral palsy increased 4fold for infants in the ninety-seventh percentile of birth weight and 16 times for male infants in the third percentile $(25,27)$. Recent studies in mice suggest that the differences between males and females in signaling pathways that regulate apoptosis may at least partly explain this epidemiological observation. Males may be more vulnerable to glutamate-mediated excitotoxicity leading to poly [ADP-ribose] polymerase (PARP)-1 activation, which causes apoptosis. One possible clue to the mechanism was the finding that levels of the energy intermediate $\mathrm{NAD}+$, which is consumed by PARP-1 activation, were reduced more in males than in females (28). Finally, we compared the relationship of brain damage (defined by $\mathrm{MRI}$ and clinical contributors), and found that children of lower gestational age and lower birth weight had a $90 \%$ greater chance of developing severe brain damage.

\section{References}

1. Perlman JM. Summary proceedings from the neurology group on hypoxic-ischemic encephalopathy. Pediatrics 2006; 117: S28-S33, doi: 10.1542/peds.2005-2425.

2. Graham EM, Ruis KA, Hartman AL, Northington FJ, Fox $\mathrm{HE}$. A systematic review of the role of intrapartum hypoxiaischemia in the causation of neonatal encephalopathy. $A m \mathrm{~J}$ Obstet Gynecol 2008; 199: 587-595, doi: 10.1016/j.ajog. 2008.06.094.

3. Jensen FE. Developmental factors regulating susceptibility to perinatal brain injury and seizures. Curr Opin Pediatr 2006; 18: 628-633, doi: 10.1097/MOP.0b013e328010c536.

4. Reed JC. Mechanisms of apoptosis. Am J Pathol 2000; 157: 1415-1430, doi: 10.1016/S0002-9440(10)64779-7.

5. Blomgren $\mathrm{K}$, Hagberg $\mathrm{H}$. Free radicals, mitochondria, and hypoxia-ischemia in the developing brain. Free Radic Biol Med 2006; 40: 388-397, doi: 10.1016/j.freeradbiomed. 2005.08.040.

6. Haynes RL, Baud O, Li J, Kinney HC, Volpe JJ, Folkerth DR. Oxidative and nitrative injury in periventricular leukomalacia: a review. Brain Pathol 2005; 15: 225-233, doi: 10.1111/j.1750-3639.2005.tb00525.x

7. Khwaja O, Volpe JJ. Pathogenesis of cerebral white matter injury of prematurity. Arch Dis Child Fetal Neonatal Ed 2008; 93: F153-F161, doi: 10.1136/adc.2006.108837.

8. Albrecht EW, Stegeman CA, Heeringa $P$, Henning $\mathrm{RH}$, van Goor $\mathrm{H}$. Protective role of endothelial nitric oxide synthase.
This is the first extensive analysis of 8 polymorphisms of NOS3 gene and their association with a large number of demographic and clinical contributors. This study not only confirmed genotype and haplotype associations of NOS3 gene polymorphism with the development of HIE but also showed their association with intensity of brain damage and severity of clinical symptoms of affected children

\section{Acknowledgments}

We are grateful to our medical and nursing colleagues and the children and their parents who agreed to take part in this study. The authors wish to thank Dr. Predrag Latkovich from Northern Montana Hospital, USA, for his assistance and for excellent and constructive comments on the manuscript prior to publication. Research supported by the Croatian Ministry of Science, Education and Sports (project No. 216-1080315-0293).
J Pathol 2003; 199: 8-17, doi: 10.1002/path.1250.

9. Baier RJ. Genetics of perinatal brain injury in the preterm infant. Front Biosci 2006; 11: 1371-1387, doi: 10.2741/1890.

10. Casas JP, Cavalleri GL, Bautista LE, Smeeth L, Humphries $\mathrm{SE}$, Hingorani AD. Endothelial nitric oxide synthase gene polymorphisms and cardiovascular disease: a HuGE review. Am J Epidemiol 2006; 164: 921-935, doi: 10.1093/aje/kwj302.

11. Howard TD, Giles WH, Xu J, Wozniak MA, Malarcher AM, Lange LA, et al. Promoter polymorphisms in the nitric oxide synthase 3 gene are associated with ischemic stroke susceptibility in young black women. Stroke 2005; 36 : 1848-1851, doi: 10.1161/01.STR.0000177978.97428.53.

12. Kuzmanić Samija R, Primorac D, Resić B, Lozić B, Krzelj V, Tomasović M, et al. Association of NOS3 tag polymorphisms with hypoxic-ischemic encephalopathy. Croat Med J 2011; 52: 396-402, doi: 10.3325/cmj.2011.52.396

13. Sarnat HB, Sarnat MS. Neonatal encephalopathy following fetal distress. A clinical and electroencephalographic study. Arch Neurol 1976; 33: 696-705, doi: 10.1001/archneur. 1976.00500100030012.

14. Weisglas-Kuperus N, Baerts W, Fetter WP, Sauer PJ. Neonatal cerebral ultrasound, neonatal neurology and perinatal conditions as predictors of neurodevelopmental outcome in very low birthweight infants. Early Hum Dev 1992; 31: 131-148, doi: 10.1016/0378-3782(92)90040-N.

15. Benjak V, Culjat M, Pavlovic M, Kostovic-Srzentic M. 
Changes of the corpus callosum in children who suffered perinatal injury of the periventricular crossroads of pathways. Coll Antropol 2008; 32 (Suppl 1): 25-29.

16. Jovanov-Milosevic N, Culjat M, Kostovic I. Growth of the human corpus callosum: modular and laminar morphogenetic zones. Front Neuroanat 2009; 3: 6.

17. Gauderman WJ. Sample size requirements for matched case-control studies of gene-environment interaction. Stat Med 2002; 21: 35-50, doi: 10.1002/sim.973.

18. Kapitanovic Vidak H, Catela Ivkovic T, Jokic M, Spaventi R, Kapitanovic $\mathrm{S}$. The association between proinflammatory cytokine polymorphisms and cerebral palsy in very preterm infants. Cytokine 2012; 58: 57-64, doi: 10.1016/j.cyto.2011. 12.018.

19. Nelson KB, Dambrosia JM, lovannisci DM, Cheng S, Grether JK, Lammer E. Genetic polymorphisms and cerebral palsy in very preterm infants. Pediatr Res 2005; 57: 494-499, doi: 10.1203/01.PDR.0000156477.00386.E7.

20. Wang J, Dudley D, Wang XL. Haplotype-specific effects on endothelial NO synthase promoter efficiency: modifiable by cigarette smoking. Arterioscler Thromb Vasc Biol 2002; 22: e1-e4, doi: 10.1161/01.ATV.0000016248.51577.1F.

21. Chorley BN, Wang $X$, Campbell MR, Pittman GS, Noureddine MA, Bell DA. Discovery and verification of functional single nucleotide polymorphisms in regulatory genomic regions: current and developing technologies. Mutat Res 2008; 659: 147-157, doi: 10.1016/j.mrrev. 2008.05.001.
22. Yoon $\mathrm{Y}$, Song J, Hong SH, Kim JQ. Plasma nitric oxide concentrations and nitric oxide synthase gene polymorphisms in coronary artery disease. Clin Chem 2000; 46: 16261630.

23. Chen W, Srinivasan SR, Li S, Boerwinkle E, Berenson GS. Gender-specific influence of NO synthase gene on blood pressure since childhood: the Bogalusa Heart Study. Hypertension 2004; 44: 668-673, doi: 10.1161/01.HYP. $0000145474.23750 .2 b$.

24. American Academy of Pediatrics. The Apgar score. Pediatrics 2006; 117: 1444-1447, doi: 10.1542/peds.20060325.

25. Lie KK, Groholt EK, Eskild A. Association of cerebral palsy with Apgar score in low and normal birthweight infants: population based cohort study. BMJ 2010; 341: c4990, doi: 10.1136/bmi.c4990.

26. Hintz SR, Kendrick DE, Vohr BR, Kenneth PW, Higgins RD. Gender differences in neurodevelopmental outcomes among extremely preterm, extremely-low-birthweight infants. Acta Paediatr 2006; 95: 1239-1248, doi: 10.1080/ 08035250600599727.

27. Thorngren-Jerneck K, Herbst A. Perinatal factors associated with cerebral palsy in children born in Sweden. Obstet Gynecol 2006; 108: 1499-1505, doi: 10.1097/ 01.AOG.0000247174.27979.6b.

28. Johnston MV, Hagberg $\mathrm{H}$. Sex and the pathogenesis of cerebral palsy. Dev Med Child Neurol 2007; 49: 74-78, doi: 10.1017/S0012162207000199.x. 